\title{
Association of Microalbuminuria with Metabolic Syndrome among Aged Population
}

\author{
Xiao-Hong Li, ${ }^{1}$ Hai-Yan Lin, ${ }^{1}$ Shu-Hua Wang, ${ }^{1}$ Li-Ying Guan, ${ }^{1}$ and Yi-Bing Wang ${ }^{2}$ \\ ${ }^{1}$ Health Management Center, Shandong Provincial Hospital Affiliated to Shandong University, Jinan, Shandong 250021, China \\ ${ }^{2}$ Department of Burn and Plastic Surgery, Shandong Provincial Hospital Affiliated to Shandong University, Jinan, \\ Shandong 250021, China \\ Correspondence should be addressed to Yi-Bing Wang; wyb0616@163.com
}

Received 31 January 2016; Revised 29 March 2016; Accepted 5 April 2016

Academic Editor: Francesco Perticone

Copyright ( 92016 Xiao-Hong Li et al. This is an open access article distributed under the Creative Commons Attribution License, which permits unrestricted use, distribution, and reproduction in any medium, provided the original work is properly cited.

\begin{abstract}
Background. The impact of the various components of metabolic syndrome (MetS) on chronic kidney disease has been conflicting. We aim to investigate the association between MetS and microalbuminuria and identify the major contributing components of MetS that result in microalbuminuria in the Chinese aged population. Methods. A total of 674 adults aged 55-98 years (males: 266; mean age: $66.5 \pm 7.5$ years) were studied. MetS was defined by the 2004 Chinese Diabetes Society criteria and microalbuminuria by urine albumin-creatinine ratio (UACR) $\geq 3 \mathrm{mg} / \mathrm{mmoL}$. Results. The prevalence of microalbuminuria was gradually increased with increasing number of MetS components $(P<0.05)$. In multivariate regression, after adjusting for age and sex, MetS was the strongest correlate of microalbuminuria $(\mathrm{OR}=1.781,95 \% \mathrm{CI}=1.226-2.587 ; P<0.05)$ followed by the fasting plasma glucose (FPG) $(\mathrm{OR}=1.217,95 \% \mathrm{CI}=1.044-1.092 ; P<0.05)$, systolic blood pressure $(\mathrm{SBP})(\mathrm{OR}=1.011,95 \% \mathrm{CI}=1.107-1.338 ; P<0.05)$, and high-density lipoprotein cholesterol (HDL-C) $(\mathrm{OR}=0.576,95 \% \mathrm{CI}=0.348-0.953 ; P<0.05)$. Conclusions. MetS is independently associated with microalbuminuria in the Chinese aged population. Elevated FPG is the most predominant component of metabolic syndrome associated with microalbuminuria followed by elevated SBP and reduced HDL-C.
\end{abstract}

\section{Introduction}

Microalbuminuria, defined as a urine albumin-creatinine ratio (UACR) of $\geq 2 \mathrm{~g} / \mathrm{mmoL}$, originally has been used as an early warning sign of chronic kidney disease and diabetic nephropathy [1]. Additionally, it has been known as a useful predictor of cardiovascular events in adults [2-4]. In light of these reports, it has been suggested that microalbuminuria screening should be added to the assessment of the cardiovascular disease risk profile, in addition to lipids and blood pressure $[5,6]$. Recently, it has been declared that obesity was associated with increasing risk of renal injury [7].

Metabolic syndrome (MetS) is a clustering of metabolic abnormalities characterized by obesity, hypertension, dyslipidemia, and glucose intolerance that collectively increases the risk of diabetes mellitus, cardiovascular disease, stroke, and overall mortality $[8,9]$. Epidemiologic studies suggested an independent association between MetS and microalbuminuria. However, studies on the association between various components of MetS and microalbuminuria, to some extent, were conflicting. Chen et al. [10], for example, reported a positive association between elevated BP, reduced HDL-C, elevated TG, and microalbuminuria in a cross-sectional study of US adults (aged $\geq 20$ years). However, another national survey of 5659 adults aged 20-80 years in the US [11] revealed that among all MetS components BP and FPG demonstrated a strong association with microalbuminuria. Thus, further supporting evidence is needed.

In the present study, 674 Chinese people aged 55-98 years were observed to evaluate the association of MetS with microalbuminuria in a cross-sectional study. We aimed to investigate whether there is an independent association for MetS and microalbuminuria, as well as determine the critical elements of MetS that correlated with microalbuminuria in the Chinese aged population. 


\section{Methods}

2.1. Study Subjects. The data for this study was an electronic record of the enterprise population who underwent annual examinations between January and April of 2015 at the Health Management Center of Shandong Provincial Hospital affiliated to Shandong University, China. The electronic record form consisted of participants' general files (register code, name, age, sex, and register date), anthropometric files (body height, body weight, and blood pressure), laboratory files (blood and urine tests results), and other files (personal history of disease). With the exclusion of subjects of age below 55 years and subjects with missing data on UACR levels and body mass index (BMI), there were 674 subjects included in our analysis.

2.2. Study Measurement. The physical examination comprised blood pressure (BP) and anthropometric measurements, including height, weight, and BMI. BMI was calculated as weight $(\mathrm{kg})$ divided by height $(\mathrm{m})^{2}$. Well-trained nurses measured BP two times in the left arm of seated participants using an automated electronic device (OMRON model HEM-752 Fuzzy, Omron Company, Dalian, China) after 5minute sitting. The average of the two readings was calculated to determine the reported BP. The venous blood samples were drawn after 12-hour overnight fasting for examining fasting plasma glucose (FPG), serum creatinine, triglyceride (TG), total cholesterol (TC), high-density lipoprotein cholesterol (HDL-C), and low-density lipoprotein cholesterol (LDL-C). Fasting 1st morning void urine specimens were obtained for determination of urinary albumin and creatinine. Urine albumin was measured using an immunoturbidimetric assay and urine creatinine using a kinetic assay using the Jaffe creatinine method. Elevated UACR in the current study was defined as UACR $\geq 3 \mathrm{mg} / \mathrm{mmoL}$ [12]. Glomerular filtration was calculated using the modified Schwartz equation: estimated glomerular filtration rate $(\mathrm{eGFR})\left(\mathrm{mL} / \mathrm{min} / 1.73 \mathrm{~m}^{2}\right)=$ $(0.413 \times$ height in $\mathrm{cm}) /$ serum creatinine in $\mathrm{mg} / \mathrm{dL}[13,14]$.

2.3. Diagnosis of MetS. According to the 2004 Chinese Diabetes Society established criteria, the MetS was defined as having any three or more of the following factors: (a) overweight and/or obesity (BMI $\geq 25.0 \mathrm{~kg} / \mathrm{m}^{2}$ ), (b) raised FPG $(\geq 6.1 \mathrm{mmol} / \mathrm{L})$ or previous diagnosis with type 2 diabetes and taking antiglycemic medication, (c) raised BP $(\geq 140 / 90 \mathrm{mmHg}$ ) or previous diagnosis with hypertension and taking antihypertensive medication, (d) raised TG $(\geq 1.7 \mathrm{mmol} / \mathrm{L})$, and/or (e) reduced HDL-C $(<0.9 \mathrm{mmol} / \mathrm{L}$ in men and $<1.0 \mathrm{mmol} / \mathrm{L}$ in women) [15].

2.4. Statistical Analysis. Data analysis was performed by using SPSS 19.0 for Windows. The distribution of the different variables was examined and the appropriate parametric or nonparametric test was applied. Data are expressed as mean or percent and standard error. Variables with skewed distribution are expressed as geometric mean (95\% confidence interval). Student's $t$-test was used to evaluate differences in mean and chi-square test to evaluate differences in proportions.

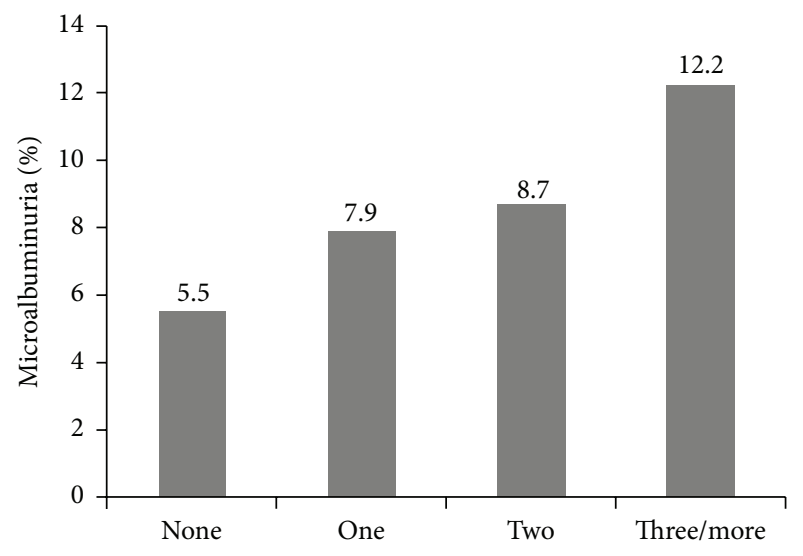

FIGURE 1: Prevalence of microalbuminuria according to the number of metabolic syndrome components.

The study participants were divided into four groups according to the number of components of MetS. Anthropometric and laboratory features in each group were described, and $P_{\text {trend }}$ across the groups was tested using linear regression analysis and Cochran-Mantel-Haenszel (CMH) for continuous and categorical variables, respectively.

A multivariate binary logistic regression model was used to find independent correlates of microalbuminuria. Adjusted odds ratios (OR) and 95\% confidence intervals (95\% CI) were calculated. Throughout analysis, $P$ value $<0.05$ was considered statistically significant.

\section{Results}

3.1. Subjects' Anthropometric and Metabolic Characteristics by the Presence of MetS. The prevalence of MetS in the study was $31.2 \%$ for men and $28.4 \%$ for women. Anthropometric and metabolic characteristics of a total of 674 subjects (266 males and 408 females) are shown in Table 1 . Mean age was $66.5 \pm 7.5$ years with $52.2 \%$ of $55-64$ years and $47.8 \%$ of over 65 years. Compared to the subjects without MetS, participants with MetS had significant increases (except for total cholesterol) in age, BMI, systolic blood pressure (SBP), diastolic blood pressure (DBP), FPG, TG, LDL-C, and UACR levels $(P<$ $0.05)$. There were also significant decreases in HDL-C and eGFR for those with MetS $(P<0.05)$.

3.2. Subjects' Anthropometric and Metabolic Characteristics by the Number of Components of MetS. The anthropometric and metabolic characteristics for participants with none $(n=134$, NMS), one ( $n=158, \mathrm{MS} 1)$, two $(n=183, \mathrm{MS} 2)$, and three or more $(n=199$, MetS) components of MetS are shown in Table 2. With the number of MetS components, there were significant increases in age, BMI, SBP, DBP, FPG, TC, TG, LDL-C, and UACR levels $\left(P_{\text {trend }}<0.0001\right)$, whereas HDL-C and eGFR decreased $\left(P_{\text {trend }}<0.0001\right)$. Figure 1 shows that the prevalence of microalbuminuria was significantly increased $(5.5,7.9,8.7$, and $12.2 \%$, resp.) with the number of components of MetS ( $P$ values for linear trend $<0.05)$. 
TABLE 1: Characteristics among participants with and without metabolic syndrome.

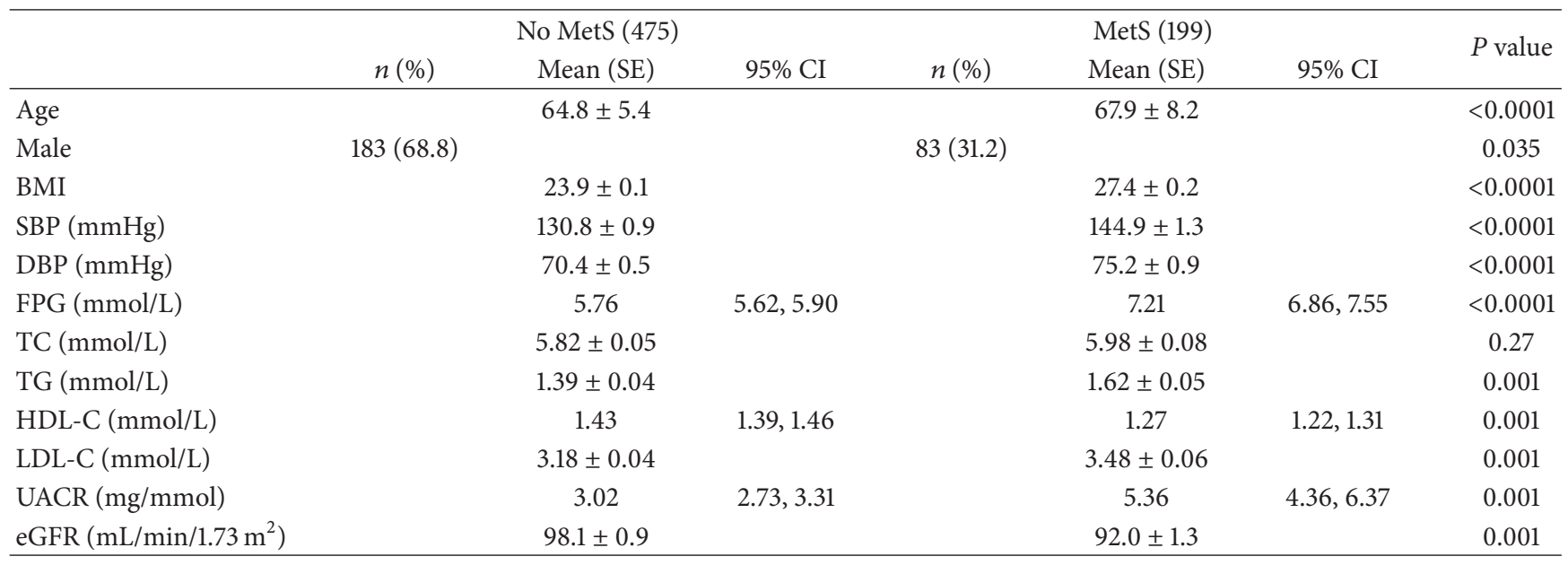

Data were means \pm SD or medians (interquartile range) for skewed variables or proportions for categorical variables. Student's $t$-test was used to evaluate differences in mean and chi-square test to evaluate differences in proportions.

MetS: metabolic syndrome; SBP: systolic blood pressure; DBP: diastolic blood pressure; BMI: body mass index; FPG: fasting plasma glucose; TG: triglyceride; TC: total cholesterol; HDL-C: high-density lipoprotein cholesterol; LDL-C: low-density lipoprotein cholesterol; UACR: urinary albumin-creatinine ratio; eGFR: estimated glomerular filtration rate.

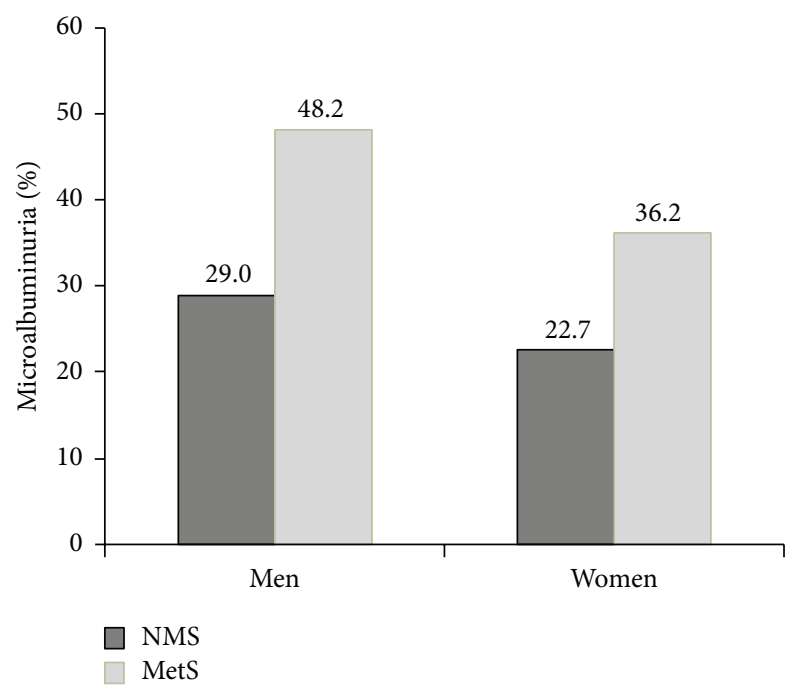

FIGURE 2: Prevalence of microalbuminuria according to metabolic syndrome.

3.3. Independent Correlates of Microalbuminuria. The prevalence of microalbuminuria was $35.7 \%$ and $31.6 \%$ for men and women, respectively. Figure 2 shows the prevalence of microalbuminuria in MetS group. In men, the prevalence of microalbuminuria was $48.2 \%$ in those with MetS and $29.0 \%$ in those without MetS $(P<0.05)$. In women, $36.2 \%$ of those with MetS had microalbuminuria, whereas $22.7 \%$ of those without MetS had microalbuminuria $(P<0.05)$.

Compared with those without microalbuminuria, the participants with microalbuminuria were older and had higher levels of SBP and FPG and lower levels of HDL-C and eGFR $(P<0.05)$ (Table 3). Based on these findings, SBP, FPG, HDL-C, eGFR, and MetS were used as potential correlates of microalbuminuria in a multivariate binary logistic regression model (Table 4). After adjusting for age and sex, MetS emerged as the strongest correlate of microalbuminuria (OR $=1.781,95 \% \mathrm{CI}=1.226-2.587 ; P=0.002)$, followed by FPG $(\mathrm{OR}=1.217,95 \% \mathrm{CI}=1.044-1.092 ; P<0.0001)$, SBP $(\mathrm{OR}=$ $1.011,95 \% \mathrm{CI}=1.107-1.338 ; P=0.014)$, and HDL-C $(\mathrm{OR}=$ $0.576,95 \% \mathrm{CI}=0.348-0.953 ; P=0.032)$.

\section{Discussion}

It has been well documented that MetS is highly prevalent worldwide, especially in affluent countries [16-18]. Several previous studies have also reported the prevalence of MetS in the Chinese population. For example, the report in 2005 by $\mathrm{Gu}$ et al. based on a cross-sectional survey in a nationally representative sample of Chinese adults aged 35-74 years reported that the age-standardised prevalence of MetS was $9.8 \%$ in men and $17.8 \%$ in women [19]. In this survey, MetS was defined according to guidelines from the US National Cholesterol Education Program. Another report of 16,442 Chinese adults (aged $\geq 18$ years) in 2010 indicated that the prevalence of MetS defined by the International Diabetes Federation (IDF) criteria was $23.2 \%(24.5 \%$ in men and $22.7 \%$ in women) [20]. In our study, the prevalence of MetS was $29.5 \%$ (31.2\% in men and $28.4 \%$ in women). Although the reports differed in the study design and the targeted populations sampled, the higher prevalence of MetS in the current study, in conjunction with the higher prevalence of $34.1 \%$ (37.8\% in men and 32.2\% in women) reported in 2014 [21], as compared to the study in 2005 by Gu et al., may truly indicate a rapidly rising trend of MetS among the Chinese population over the 10-year period.

In the current study, the prevalence of microalbuminuria increased significantly with increasing numbers of MetS components after the participants were categorized according 


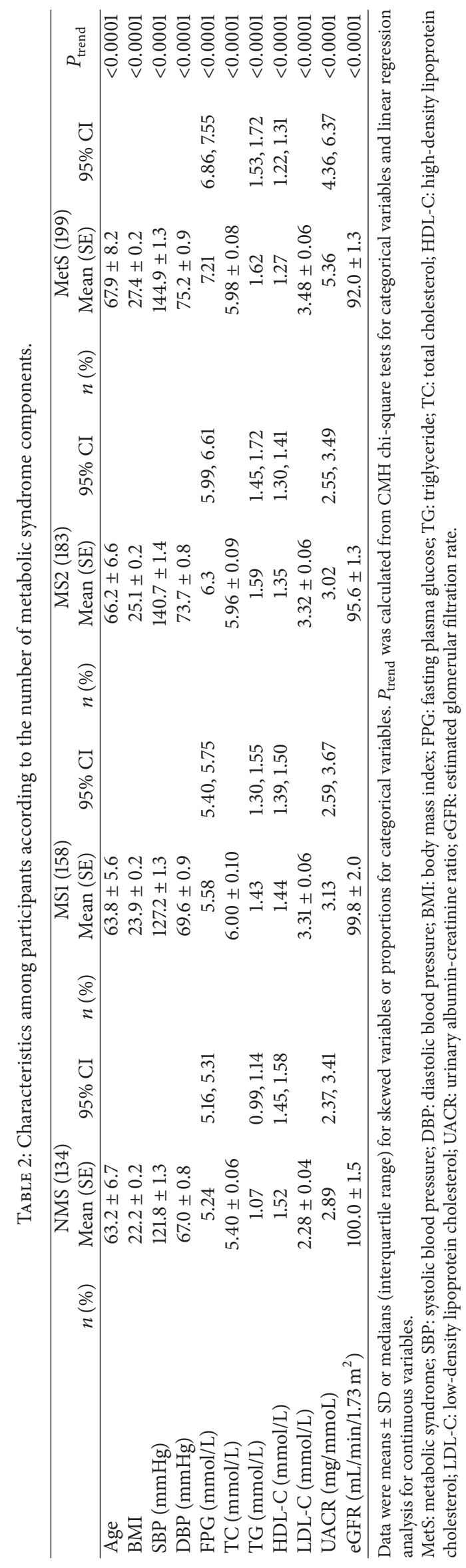


TABLE 3: Characteristics among participants with and without elevated UACR.

\begin{tabular}{|c|c|c|c|c|c|c|c|}
\hline & \multicolumn{3}{|c|}{ UACR < $3 \mathrm{mg} / \mathrm{mmol}(450)$} & \multicolumn{3}{|c|}{ UACR $\geq 3 \mathrm{mg} / \mathrm{mmol}(224)$} & \multirow{2}{*}{$P$ value } \\
\hline & $n(\%)$ & Mean (SE) & $95 \% \mathrm{CI}$ & $n(\%)$ & Mean (SE) & $95 \%$ CI & \\
\hline Age & & $64.2 \pm 6.3$ & & & $68.4 \pm 7.6$ & & $<0.0001$ \\
\hline BMI & & $25.0 \pm 0.2$ & & & $25.1 \pm 0.2$ & & 0.427 \\
\hline $\mathrm{SBP}(\mathrm{mmHg})$ & & $132.4 \pm 0.8$ & & & $140.7 \pm 1.5$ & & $<0.0001$ \\
\hline DBP (mmHg) & & $71.5 \pm 0.5$ & & & $72.7 \pm 0.9$ & & 0.317 \\
\hline FPG (mmol/L) & & 5.8 & $5.69,5.98$ & & 6.7 & $6.40,7.07$ & $<0.0001$ \\
\hline TC (mmol/L) & & $5.9 \pm 0.1$ & & & $5.8 \pm 0.1$ & & 0.279 \\
\hline $\mathrm{TG}(\mathrm{mmol} / \mathrm{L})$ & & $1.43 \pm 0.04$ & & & $1.51 \pm 0.05$ & & 0.087 \\
\hline $\mathrm{HDL}-\mathrm{C}(\mathrm{mmol} / \mathrm{L})$ & & 1.41 & $1.38,1.45$ & & 1.35 & $1.30,1.39$ & 0.009 \\
\hline LDL-C (mmol/L) & & $3.28 \pm 0.04$ & & & $3.22 \pm 0.05$ & & 0.633 \\
\hline $\mathrm{eGFR}\left(\mathrm{mL} / \mathrm{min} / 1.73 \mathrm{~m}^{2}\right)$ & & $97.8 \pm 0.9$ & & & $94.5 \pm 1.3$ & & 0.005 \\
\hline MetS (\%) & $117(26.0)$ & & & $82(36.6)$ & & & $<0.0001$ \\
\hline
\end{tabular}

Elevated UACR was defined as a UACR $\geq 3 \mathrm{mg} / \mathrm{mmol}$. Data were means \pm SD or medians (interquartile range) for skewed variables or proportions for categorical variables. Student's $t$-test was used to evaluate differences in mean and chi-square test to evaluate differences in proportions.

MetS: metabolic syndrome; SBP: systolic blood pressure; DBP: diastolic blood pressure; BMI: body mass index; FPG: fasting plasma glucose; TG: triglyceride; TC: total cholesterol; HDL-C: high-density lipoprotein cholesterol; LDL-C: low-density lipoprotein cholesterol; UACR: urinary albumin-creatinine ratio; eGFR: estimated glomerular filtration rate.

TABLE 4: Binary logistic regression to find independent correlates of microalbuminuria.

\begin{tabular}{|c|c|c|c|c|}
\hline & \multicolumn{2}{|c|}{ Unadjusted } & \multicolumn{2}{|c|}{ Age- and gender-adjusted } \\
\hline & OR $(95 \% \mathrm{CI})$ & $P$ value & OR $(95 \% \mathrm{CI})$ & $P$ value \\
\hline SBP & $1.019(1.010,1.027)$ & $<0.0001$ & $1.011(1.107,1.338)$ & 0.014 \\
\hline FPG & $1.257(1.142,1.383)$ & $<0.0001$ & $1.217(1.044,1.092)$ & $<0.0001$ \\
\hline HDL-C & $0.556(0.348,0.886)$ & 0.013 & $0.576(0.348,0.953)$ & 0.032 \\
\hline eGFR & $0.986(0.976,0.996)$ & 0.005 & $0.993(0.981,1.005)$ & 0.252 \\
\hline MetS & $2.061(1.437,2.956)$ & $<0.0001$ & $1.781(1.226,2.587)$ & 0.002 \\
\hline
\end{tabular}

MetS: metabolic syndrome; SBP: systolic blood pressure; FPG: fasting plasma glucose; HDL-C: high-density lipoprotein cholesterol; eGFR: estimated glomerular filtration rate.

to the number of MetS components they had. The same result was obtained in a large study on 2,321 subjects in Japan [22]. The level of UACR was increased significantly even in the subjects with 1 or 2 components of metabolic syndrome. Therefore, it is suggested that earlier intervention for metabolic syndrome should be started to prevent the progression of chronic kidney disease.

The association between MetS and microalbuminuria has been shown in many studies [11, 23-27]. A study of Japanese people aged 40 to 87 years showed that, compared with those without MetS, those with MetS had a 1.99-fold (95\% CI: 1.49 to 2.66) higher risk of microalbuminuria after adjusting for age and sex [22]. Another study of Korean adults aged 19 years or older reported that, compared with those without MetS, men and women with MetS had 3and 2.79-fold higher risk of microalbuminuria, respectively, after adjusting for covariates [28]. However, the relationship between MetS and microalbuminuria has not been well elucidated in the Chinese aged population yet. In our study, we found that MetS was an independent and strong correlate of microalbuminuria in the aged population. Furthermore, MetS increased the risk of microalbuminuria 1.781-fold (95\% CI: 1.226 to 2.587 ) after adjusting for age and gender.
Studies have yielded mixed results for the association between various fractions of MetS and microalbuminuria. For example, $\mathrm{Hao}$ et al. reported a positive association between high FPG, high BP, obesity, and microalbuminuria in a cross-sectional study of 2321 adults [22]. A study conducted on patients undergoing coronary angiography revealed among the components of MetS high FPG and lower HDL-C related to microalbuminuria [29]. Based on the impact of the various components of MetS on chronic kidney disease, the most recent study presented the concept of dividing all five components of MetS into a critical arm including elevated BP, reduced HDL-C, and elevated FPG and a noncritical arm including elevated TG and obesity [30]. Therefore, the impact of the components of MetS on microalbuminuria should be interpreted judiciously. Our results showed that elevated FPG was the most predominant component of metabolic syndrome associated with microalbuminuria followed by elevated SBP and reduced HDL-C. This result indicates that elevated FPB and SBP and lower HDL-C are likely to be critical components that lead a substantial number of subjects to the prestage of metabolic syndrome in the Chinese aged population. 
Despite the previous reports, in our study, elevated SBP, not DBP, was associated with microalbuminuria. Indirect sphygmomanometric BP measurement is an established way of diagnosing and monitoring hypertension but overestimates the frequency of elevated DBP in the aged population [31]. Thus, the weaker association between the diastolic component of the arterial pressure and microalbuminuria in our study may be explained in part by the inclusion of subjects falsely labeled as hypertensive by the cuff sphygmomanometer in the aged population.

This study has some limitations. First, our population number was relatively small. Thus, prospective studies of a large sample should be conducted to verify the relationship between MetS and microalbuminuria. Second, the crosssectional study design makes it hard to infer causality between MetS and microalbuminuria. Third, instead of waist circumference, BMI was used in the definition of MetS due to lack of corresponding data. This lack of data could have led to misestimation of the prevalence of MetS. Finally, ACR was determined on the basis of a single urine collection that participants were asked to collect in the morning of their visit. However, the high diagnostic accuracy of a single urine collection should minimise the uncertainty associated with day-to-day differences in the degree of albumin excretion [32].

In conclusion, the present study revealed a strong relationship between microalbuminuria and metabolic syndrome in the aged population in China. More comprehensive and intensive management of metabolic syndrome at its early stage should be started to prevent the progression of renal injury and cardiovascular complication.

\section{Competing Interests}

The authors certify that there are no competing interests regarding the publication of this paper.

\section{Acknowledgments}

The authors thank all doctors, nurses, and other staff members of the Health Management Center, Shandong Provincial Hospital affiliated to Shandong University, and Mr. Zhao Kai (Network Center) for his involvement in the project.

\section{References}

[1] H. C. Gerstein, J. F. E. Mann, J. Pogue et al., "Prevalence and determinants of microalbuminuria in high-risk diabetic and nondiabetic patients in the heart outcomes prevention evaluation study," Diabetes Care, vol. 23, supplement 2, pp. B35B39, 2000.

[2] M. E. Widlansky, N. Gokce, J. F. Keaney Jr., and J. A. Vita, “The clinical implications of endothelial dysfunction," Journal of the American College of Cardiology, vol. 42, no. 7, pp. 1149-1160, 2003.

[3] J. J. Cao, J. I. Barzilay, D. Peterson et al., "The association of microalbuminuria with clinical cardiovascular disease and subclinical atherosclerosis in the elderly: the Cardiovascular Health study," Atherosclerosis, vol. 187, no. 2, pp. 372-377, 2006.
[4] D. Dutta, S. Choudhuri, S. A. Mondal, S. Mukherjee, and S. Chowdhury, "Urinary albumin: creatinine ratio predicts prediabetes progression to diabetes and reversal to normoglycemia: role of associated insulin resistance, inflammatory cytokines and low vitamin D," Journal of Diabetes, vol. 6, no. 4, pp. 316322, 2014.

[5] R. Pedrinelli, G. Dell'Omo, G. Penno, and M. Mariani, "Nondiabetic microalbuminuria, endothelial dysfunction and cardiovascular disease," Vascular Medicine, vol. 6, no. 4, pp. 257264, 2001.

[6] H. C. Gerstein, J. F. E. Mann, Q. Yi et al., "Albuminuria and risk of cardiovascular events, death, and heart failure in diabetic and nondiabetic individuals," The Journal of the American Medical Association, vol. 286, no. 4, pp. 421-426, 2001.

[7] N. Xiao, T. M. Jenkins, E. Nehus et al., "Kidney function in severely obese adolescents undergoing bariatric surgery," Obesity, vol. 22, no. 11, pp. 2319-2325, 2014.

[8] D. E. Laaksonen, H.-M. Lakka, L. K. Niskanen, G. A. Kaplan, J. T. Salonen, and T. A. Lakka, "Metabolic syndrome and development of diabetes mellitus: application and validation of recently suggested definitions of the metabolic syndrome in a prospective cohort study," American Journal of Epidemiology, vol. 156, no. 11, pp. 1070-1077, 2002.

[9] B. Isomaa, P. Almgren, T. Tuomi et al., "Cardiovascular morbidity and mortality associated with the metabolic syndrome," Diabetes Care, vol. 24, no. 4, pp. 683-689, 2001.

[10] J. Chen, P. Muntner, L. L. Hamm et al., "The metabolic syndrome and chronic kidney disease in U.S. adults," Annals of Internal Medicine, vol. 140, no. 3, pp. 167-I39, 2004.

[11] L. Palaniappan, M. Carnethon, and S. P. Fortmann, "Association between microalbuminuria and the metabolic syndrome: NHANES III," American Journal of Hypertension, vol. 16, no. 11, pp. 952-958, 2003.

[12] E. Crowe, D. Halpin, and P. Stevens, "Early identification and management of chronic kidney disease: summary of NICE guidance," The British Medical Journal, vol. 337, Article ID a1530, 2008.

[13] G. J. Schwartz, A. Muñoz, M. F. Schneider et al., "New equations to estimate GFR in children with CKD," Journal of the American Society of Nephrology, vol. 20, no. 3, pp. 629-637, 2009.

[14] L. Selistre, V. De Souza, P. Cochat et al., "GFR estimation in adolescents and young adults," Journal of the American Society of Nephrology, vol. 23, no. 6, pp. 989-996, 2012.

[15] Society EPoMSoCD, "Recommendations provided by Chinese diabetes society on metabolic syndrome," Chinese Journal of Diabetes, vol. 12, pp. 156-161, 2004 (Chinese).

[16] E. S. Ford, "Prevalence of the metabolic syndrome defined by the international diabetes federation among adults in the U.S," Diabetes Care, vol. 28, no. 11, pp. 2745-2749, 2005.

[17] H.-M. Lakka, D. E. Laaksonen, T. A. Lakka et al., “The metabolic syndrome and total and cardiovascular disease mortality in middle-aged men," The Journal of the American Medical Association, vol. 288, no. 21, pp. 2709-2716, 2002.

[18] R. Miccoli, C. Bianchi, L. Odoguardi et al., "Prevalence of the metabolic syndrome among Italian adults according to ATP III definition," Nutrition, Metabolism and Cardiovascular Diseases, vol. 15, no. 4, pp. 250-254, 2005.

[19] D. Gu, K. Reynolds, X. Wu et al., "Prevalence of the metabolic syndrome and overweight among adults in China," The Lancet, vol. 365, no. 9468, pp. 1398-1405, 2005. 
[20] G. Li, M. de Courten, S. Jiao, and Y. Wang, "Prevalence and characteristics of the metabolic syndrome among adults in Beijing, China," Asia Pacific Journal of Clinical Nutrition, vol. 19, no. 1, pp. 98-102, 2010.

[21] X. G. Hou, C. Wang, Z. Q. Ma et al., "Optimal waist circumference cut-off values for identifying metabolic risk factors in middle-aged and elderly subjects in shandong province of China," Biomedical and Environmental Sciences, vol. 27, no. 5, pp. 353-359, 2014.

[22] Z. Hao, T. Konta, S. Takasaki et al., "The association between microalbuminuria and metabolic syndrome in the general population in Japan: the Takahata study," Internal Medicine, vol. 46, no. 7, pp. 341-346, 2007.

[23] I.-T. Lee, W.-J. Lee, C.-N. Huang, and W. H-H Sheu, "The association of low-grade inflammation, urinary albumin, and insulin resistance with metabolic syndrome in nondiabetic Taiwanese," Metabolism: Clinical and Experimental, vol. 56, no. 12, pp. 1708-1713, 2007.

[24] K. P. Klausen, H.-H. Parving, H. Scharling, and J. S. Jensen, “The association between metabolic syndrome, microalbuminuria and impaired renal function in the general population: impact on cardiovascular disease and mortality," Journal of Internal Medicine, vol. 262, no. 4, pp. 470-478, 2007.

[25] T. Ninomiya and Y. Kiyohara, "Albuminuria and chronic kidney disease in association with the metabolic syndrome," Journal of the Cardiometabolic Syndrome, vol. 2, pp. 104-107, 2007.

[26] H. S. Choi, S. H. Ryu, and K.-B. Lee, "The relationship of microalbuminuria with metabolic syndrome," Nephron Clinical Practice, vol. 104, no. 2, pp. c85-c93, 2006.

[27] K. Rowley, K. O’Dea, and J. D. Best, "Association of albuminuria and the metabolic syndrome," Current Diabetes Reports, vol. 3, no. 1, pp. 80-86, 2003.

[28] H.-O. Lee, H.-J. Bak, J.-Y. Shin, and Y.-M. Song, "Association between metabolic syndrome and microalbuminuria in Korean adults," Korean Journal of Family Medicine, vol. 36, no. 2, pp. 60-71, 2015.

[29] M. S. Anvari, M. A. Boroumand, L. Pourgholi et al., "Potential link of microalbuminuria with metabolic syndrome in patients undergoing coronary angiography," Archives of Medical Research, vol. 40, no. 5, pp. 399-405, 2009.

[30] H. Song, X. Wang, Q. Cai, W. Ding, S. Huang, and L. Zhuo, "Association of metabolic syndrome with decreased glomerular filtration rate among 75,468 chinese adults: a cross-sectional study," PLoS ONE, vol. 9, no. 11, Article ID el13450, 2014.

[31] K. M. Hla, K. A. Vokaty, and J. R. Feussner, "Overestimation of diastolic blood pressure in the elderly. Magnitude of the problem and a potential solution," Journal of the American Geriatrics Society, vol. 33, no. 10, pp. 659-663, 1985.

[32] W. Hofmann and W. G. Guder, "A diagnostic programme for quantitative analysis of proteinuria," Journal of Clinical Chemistry and Clinical Biochemistry, vol. 27, no. 9, pp. 589-600, 1989. 


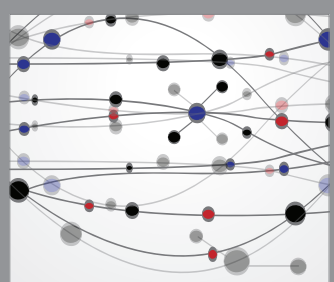

The Scientific World Journal
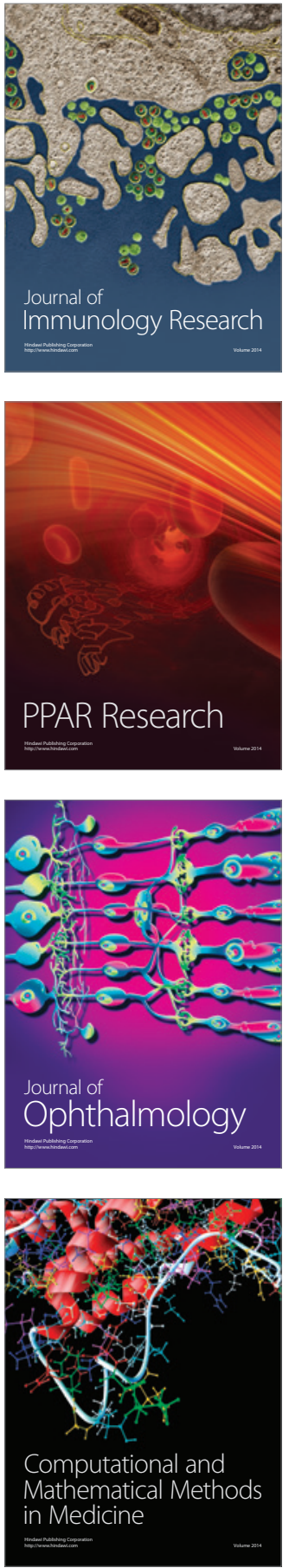

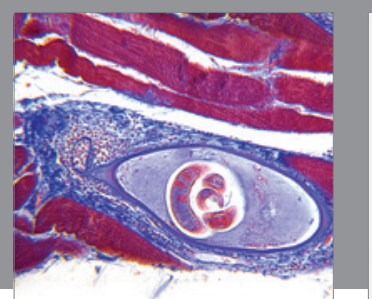

Gastroenterology Research and Practice

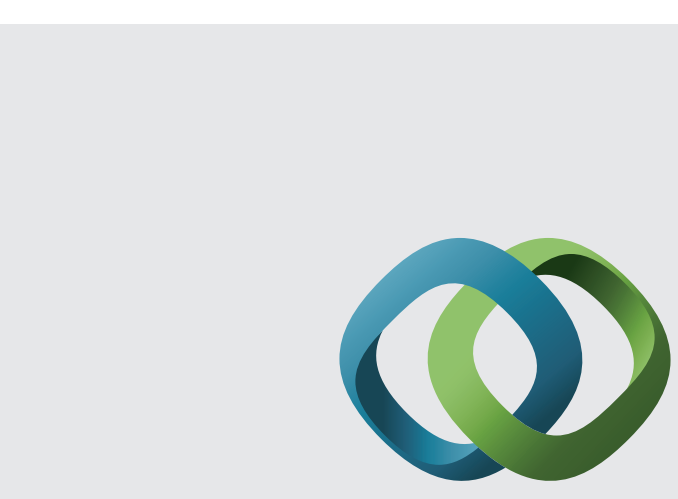

\section{Hindawi}

Submit your manuscripts at

http://www.hindawi.com
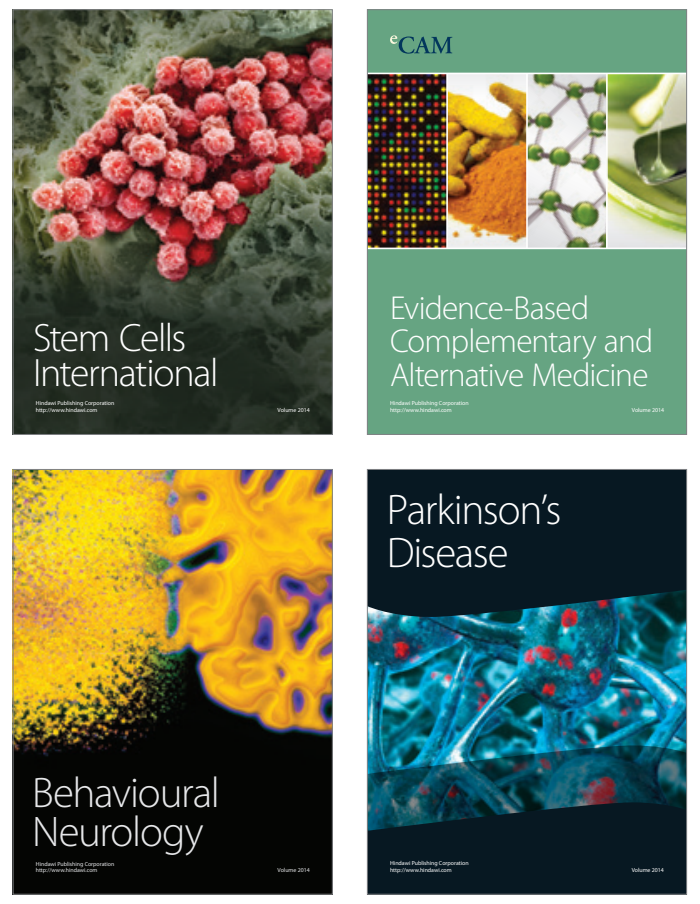
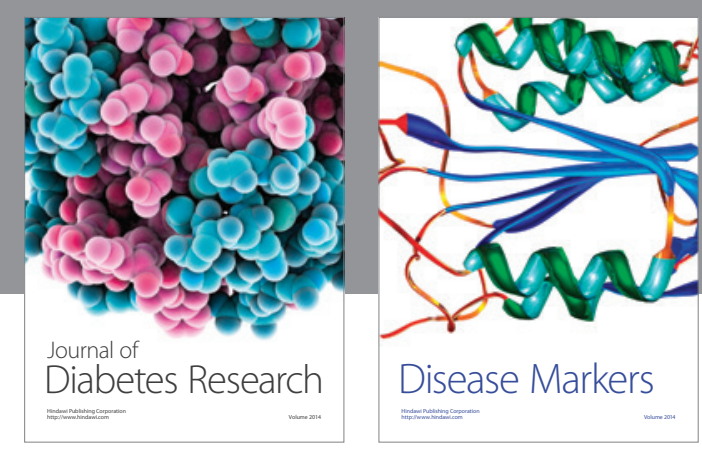

Disease Markers
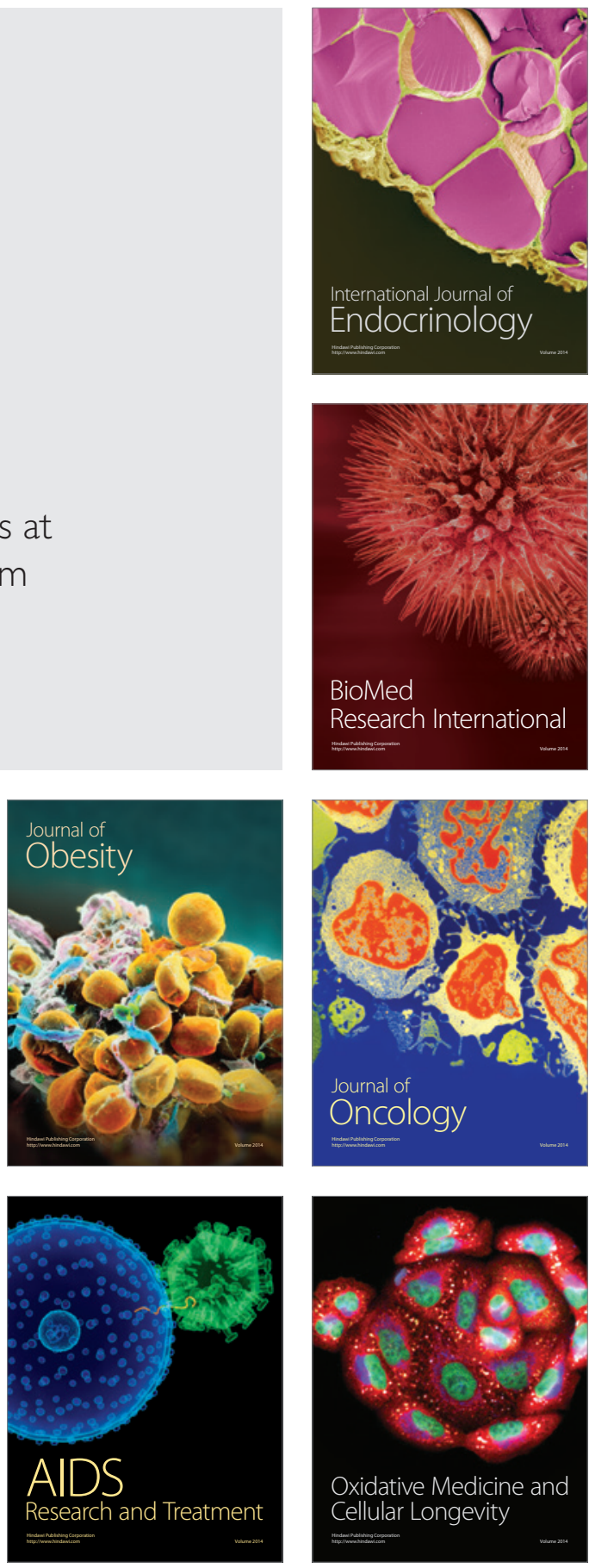